\title{
A CASSCF/CSAPT2 Study on the Reaction Pathways From HOSS to HSSO Radicals
}

\author{
Haifeng Zhang, Jiubo Cui, Weiwei Pei*, and Dongfang Wang* \\ College of Science, Jiamusi University, 154007 Jiamusi, China
}

\begin{abstract}
In this paper, we used the CASSCF/CASPT2 method to study the low-lying electronic states of the HOSS and HSSO radicals. Based on the Cs and $\mathrm{C} 1$ symmetries, the geometrical parameters, total energies, configurations, oscillator strengths, excitation energies, oscillator strengths and harmonic vibrational frequencies of the HOSS and HSSO radicals were calculated. We found that the ground state of HSSO radical with $\mathrm{C} 1$ symmetry has multi-configurational character, which leads to a significant difference of dihedral angles of HSSO radical among at our work and at references. Moreover, a transition state was given out in the pathway for the reaction from HOSS to HSSO. This transition state produces relatively high-energy barrier of $30.5 \mathrm{Kcal} / \mathrm{mol}$ corresponding to the gradual changes of the S2-H-O angle.
\end{abstract}

\section{Introduction}

Sulfur is an essential component of all living cells and has significant implications in the atmosphere and environment. In our nature, there are a large number of sulfur-rich compounds which contain hydrogen and oxygen atoms.

Through mass spectrometric techniques and ab initio calculations, Giulia et al. studied radicals, cations and anions of the species in 2007[1]. And they suggested that the cis HOSS is the most stable structure and the HSSO radical is nonplanar. Daniel et al. studied the heats of formation, atomization energies, and bond dissociation energies of the HOmSn (m, n =0-3) molecules in their theoretical calculations[2]. In 2012, through comparing the computed and experimental IR spectra for the HSSO and HOSS radicals, Hans et al. believed that the broad absorption band of HOSS radical either at 366 or at 405 $\mathrm{nm}$, which induced the $\mathrm{H}$ shift and leaded to the isomeric radical HSSO. And this photoisomerization was reversible at $\lambda=546 \mathrm{~nm}[3]$.

However, they did not give any available information for the excited states of these species. As far as we known, there is a shortage of the theoretical studies on excitation spectroscopy and dissociation of the HOSS and HSSO radicals that makes us conduct a comprehensive investigation into providing accurate prediction for the excited states.

\section{Computational details}

In this work, the geometries, total energies, harmonic vibrational frequencies, vertical and adiabatic excitation energies, and oscillator strengths of the HOSS and HSSO radicals were calculated at the CASSCF[4] and CASPT2[5] levels with an applied level shift of 0.3 a.u. to prevent the intruder state problem happening, which employs the correlation-consistent valence-polarized basis set aug-cc-pV(n+d)Z, n=D,T, Q.

For each of radicals, we chose the 19 active electrons ,10 occupied orbitals and 3 added vacant orbitals as the active space. The ground states of the HOSS and HSSO radicals are presented as $12 \mathrm{~A}^{\prime \prime}$ and $12 \mathrm{~A}$ respectively. On the basis of the optimized geometries, we performed CASPT2 calculations at all CASSCF stationary points to identify the adiabatic excitation energies Ea and vertical excitation energies Ev. Using the CASPT2 energy differences, the oscillator strengths $f$ were computed by the CASSI program[6] .

To calculate the harmonic vibrational frequencies, the multi-configurational linear response (MCLR) was used. And we have tried to calculate the geometries of ground state of HSSO radical at the CASPT2 level, using the basis sets jul-cc-pV(T+d)Z, jun-cc-pV(T+d)Z, maugcc-pV $(\mathrm{T}+\mathrm{d}) \mathrm{Z}$, aug-cc-pV(T+d)Z, ano-rcc-pVTZ, and 6$311++\mathrm{G}(3 \mathrm{df}, 3 \mathrm{pd})$.

In addition to the transition states (TS) between the HOSS and HSSO radicals, our focus was also paid on reaction pathways involving the lowest state $\mathrm{D}_{0}$ of the HOSS and HSSO radicals, finding a potential energy surface of these states to examine their roles in the reaction dynamics. All the above calculations were performed by using MOLCAS 7.8 programs.

\section{Results and discussion}

\subsection{Geometries optimization}

The atom labels of HOSS and HSSO radicals were showen in Figure 1.

\footnotetext{
* Corresponding author: peiweiwei50082@126.com; jmsdxwdf2012@126.com
} 


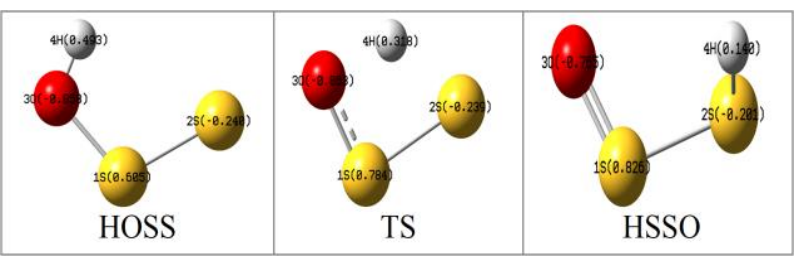

Fig.1. Atom labels with the NBO charge distribution.

Calculations were performed by means of the CASSCF and CASPT2 methods, using the aug-cc$\mathrm{pV}(\mathrm{n}+\mathrm{d}) \mathrm{Z}$ basis sets, $\mathrm{n}=\mathrm{D}, \mathrm{T}, \mathrm{Q}$. The calculated bond lengths (in $\AA$ ) and bond angle (in ${ }^{\circ}$ ) for the ground states of the HOSS and HSSO radicals are listed in Table 1a and Table $1 \mathrm{~b}$. At the same time, the geometry parameters of the 12A of TS are listed in Table 1c. And the configurations and CI coefficients for the states of HOSS and HSSO radicals are listed in Table $2 \mathrm{a}$ and $2 \mathrm{~b}$. For the $12 \mathrm{~A}$ " state of HOSS radical at the CASSCF/ aug-cc$\mathrm{pV}(\mathrm{T}+\mathrm{d}) \mathrm{Z}$ Level, the plots of some occupied orbitals and vacant orbitals in relation to electron transitions listed in Table 2 are shown in Figure 2.

Table 1a. Geometries parameters of the 2A" of HOSS.

\begin{tabular}{ccccccc}
\hline Method /Basis set & $r(\mathrm{~S}-\mathrm{S})$ & $r(\mathrm{~S}-\mathrm{O})$ & $r(\mathrm{O}-\mathrm{H})$ & $\angle \mathrm{SSO}$ & $\angle \mathrm{SOH}$ & $\angle \mathrm{HSSO}$ \\
\hline CASSCF/ aug-cc-pV(D+d)Z & 1.954 & 1.702 & 0.976 & 109.2 & 105.9 & 0 \\
CASSCF/ aug-cc-pV(T+d)Z & 1.943 & 1.669 & 0.973 & 109.3 & 106.7 & 0 \\
CASSCF/ aug-cc-pV(Q+d)Z & 1.940 & 1.665 & 0.971 & 109.3 & 106.9 & 0 \\
CASPT2/ aug-cc-pV(D+d)Z & 1.932 & 1.702 & 0.977 & 104.8 & 104.3 & 0 \\
CASPT2/ aug-cc-pV(T+d)Z & 1.914 & 1.659 & 0.972 & 109.3 & 107.2 & 0 \\
CASPT2/ aug-cc-pV(Q+d)Z & 1.907 & 1.651 & 0.970 & 106.9 & 106.2 & 0 \\
[3]UB3LYP/6-311+G(3df,3pd) & 1.922 & 1.665 & 0.969 & 109.9 & 108.8 & 0 \\
[3]UCCSD(T)-FC/cc-pVTZ & 1.934 & 1.668 & 0.970 & 108.5 & 105.7 & 0 \\
[7]UCCSD(T)/ aug-cc-pV(T+d)Z & 1.922 & 1.660 & 0.971 & 109.0 & 107.0 & 0 \\
\hline
\end{tabular}

Table 1b. Geometries parameters of the 2A of HSSO.

\begin{tabular}{ccccccc}
\hline Method /Basis set & $\mathrm{r}(\mathrm{S}-\mathrm{S})$ & $\mathrm{r}(\mathrm{S}-\mathrm{O})$ & $\mathrm{r}(\mathrm{S}-\mathrm{H})$ & $\angle \mathrm{SSO}$ & $\angle$ HSS & $\angle$ HSSO \\
\hline CASSCF/ aug-cc-pV(D+d)Z & 2.110 & 1.505 & 1.337 & 110.5 & 95.2 & 74.4 \\
CASSCF/ aug-cc-pV(T+d)Z & 2.096 & 1.483 & 1.329 & 110.7 & 96.3 & 73.3 \\
CASSCF/ aug-cc-pV(Q+d)Z & 2.093 & 1.480 & 1.329 & 110.7 & 96.4 & 73.4 \\
CASPT2/ aug-cc-pV(D+d)Z & 2.150 & 1.517 & 1.349 & 110.5 & 92.7 & 76.7 \\
CASPT2/ aug-cc-pV(T+d)Z & 2.113 & 1.495 & 1.338 & 111.5 & 94.1 & 88.9 \\
CASPT2/ aug-cc-pV(Q+d)Z & 2.116 & 1.483 & 1.337 & 111.1 & 93.2 & 80.0 \\
CASPT2/ jul-cc-pV(T+d)Z & 2.126 & 1.489 & 1.338 & 111.1 & 93.2 & 80.0 \\
CASPT2/ jun-cc-pV(T+d)Z & 2.216 & 1.488 & 1.337 & 111.1 & 93.2 & 79.5 \\
CASPT2/ maug-cc-pV(T+d)Z & 2.216 & 1.487 & 1.337 & 111.2 & 93.1 & 78.7 \\
CASPT2/ aug-cc-pVTZ & 2.113 & 1.505 & 1.342 & 110.2 & 93.6 & 88.0 \\
CASPT2/ ano-rcc-pVTZ & 2.110 & 1.500 & 1.340 & 111.2 & 94.5 & 91.8 \\
CASPT2/ 6-311++G(3df,3pd) & 2.106 & 1.490 & 1.337 & 111.2 & 94.0 & 85.6 \\
[3]UB3LYP/6-311+G(3df,3pd) & 2.117 & 1.484 & 1.374 & 110.8 & 95.8 & 63.8 \\
[3]UCCSD(T)-FC/cc-pVTZ & 2.126 & 1.495 & 1.345 & 109.8 & 93.8 & 64.0
\end{tabular}

Table 1c. Geometries parameters of the $2 \mathrm{~A}$ of TS.

\begin{tabular}{ccccccc}
\hline Basis set & $\mathrm{r}(\mathrm{S}-\mathrm{S})$ & $\mathrm{r}(\mathrm{S}-\mathrm{O})$ & $\mathrm{r}(\mathrm{S}-\mathrm{H})$ & $\angle \mathrm{SSO}$ & $\angle \mathrm{HSS}$ & $\angle \mathrm{HSSO}$ \\
\hline aug-cc-pV(D+d)Z & 2.006 & 1.559 & 1.384 & 94.0 & 62.3 & 0 \\
aug-cc-pV(T+d)Z & 2.007 & 1.559 & 1.384 & 94.0 & 62.3 & 0 \\
aug-cc-pV(Q+d)Z & 2.015 & 1.560 & 1.376 & 93.0 & 63.4 & 0 \\
\hline
\end{tabular}

At the CASSCF and CASPT2 levels, the 12A" and $12 \mathrm{~A}$ states was predicted to be the ground states of HOSS and HSSO radicals, respectively. From the Table 1 , we can see that, for the obtained geometries parameters of HOSS radical using the aug-cc-pV $(n+d) Z$ basis sets in our work, the calculated values in the CASPT2 level are closer to the values of other theoretical calculation methods than the ones in the CASSCF level.

\subsection{The calculations on Adiabatic Energies Ea and Vertical Excitation Energies Ev}

As the Table 2 shown, we also calculated the adiabatic excitation energies Ea of the low lying excited states of HOSS and HSSO radicals at the CASSCF and CASPT2 levels, in which the dynamical correlation effects were considered. 
Table 2a. Configurations, Coeff., Ea, Ev, and f for the HOSS. ${ }^{a}$

\begin{tabular}{|c|c|c|c|c|c|c|c|}
\hline \multirow{2}{*}{\multicolumn{2}{|c|}{ State }} & \multicolumn{2}{|l|}{ Configuration } & \multicolumn{2}{|c|}{$\mathrm{Ea}(\mathrm{eV})$} & \multirow{2}{*}{$\begin{array}{c}\mathrm{Ev} \\
(\mathrm{eV} / \mathrm{nm})\end{array}$} & \multirow{2}{*}{$\mathrm{f}$} \\
\hline & & Occupation & Coeff. & CASSCF & CASPT2 & & \\
\hline \multirow[t]{4}{*}{$\mathrm{CS}$} & $12 \mathrm{~A}^{\prime \prime}$ & $\begin{array}{c}\left(15 \mathrm{a}^{\prime}\right)^{2}\left(4 \mathrm{a}^{\prime \prime}\right)^{2}\left(16 \mathrm{a}^{\prime}\right)^{2} \\
\left(15 \mathrm{a}^{\prime}\right)^{2}\left(4 \mathrm{a}^{\prime \prime}\right)^{\alpha}\left(16 \mathrm{a}^{\prime}\right)^{2}\left(5 \mathrm{a}^{\prime \prime}\right)^{2}\end{array}$ & $\begin{array}{c}-0.924 \\
0.226\end{array}$ & 0 & 0 & 0 & \\
\hline & $12 \mathrm{~A}^{\prime}$ & $\begin{array}{l}\left(15 a^{\prime}\right)^{2}\left(4 a^{\prime \prime}\right)^{2}\left(16 a^{\prime}\right)^{\alpha}\left(5 a^{\prime \prime}\right)^{2} \\
\left(15 a^{\prime}\right)^{\alpha}\left(4 a^{\prime \prime}\right)^{2}\left(16 a^{\prime}\right)^{2}\left(5 a^{\prime \prime}\right)^{2}\end{array}$ & $\begin{array}{l}0.654 \\
0.288\end{array}$ & 1.87 & 1.90 & $2.23 / 556$ & $3.85 \times 10-4$ \\
\hline & $22 \mathrm{~A}^{\prime \prime}$ & $\begin{array}{l}\left(15 \mathrm{a}^{\prime}\right)^{2}\left(4 \mathrm{a}^{\prime \prime}\right)^{2}\left(16 \mathrm{a}^{\prime}\right)^{2}\left(5 \mathrm{a}^{\prime \prime}\right) \alpha \\
\left(15 \mathrm{a}^{\prime}\right)^{2}\left(4 \mathrm{a}^{\prime \prime}\right)^{\alpha}\left(16 \mathrm{a}^{\prime}\right)^{2}\left(5 \mathrm{a}^{\prime \prime}\right)^{2}\end{array}$ & $\begin{array}{l}-0.252 \\
-0.838\end{array}$ & 2.80 & 2.78 & $3.33 / 372$ & $4.8 \times 10-2$ \\
\hline & $22 \mathrm{~A}^{\prime}$ & $\left(15 a^{\prime}\right)^{2}\left(4 a^{\prime \prime}\right)^{2}\left(16 a^{\prime}\right)^{2}\left(17 a^{\prime}\right)^{\alpha}$ & 0.929 & 3.42 & 3.15 & $3.33 / 372$ & $1.3 \times 10-3$ \\
\hline \multirow[t]{4}{*}{$\mathrm{C} 1$} & $12 \mathrm{~A}$ & $(18 a)^{2}(19 a)^{2}(20 a)^{2}(21 a)^{\alpha}$ & -0.966 & 0 & 0 & 0 & \\
\hline & $22 \mathrm{~A}$ & $\begin{array}{l}(18 a)^{2}(19 a)^{2}(20 a) \alpha(21 a)^{2} \\
(18 a)^{2}(19 a)^{\alpha}(20 a)^{2}(21 a)^{2}\end{array}$ & $\begin{array}{c}-0.933 \\
0.261\end{array}$ & 1.66 & 1.50 & $2.31 / 537$ & $3.5 \times 10-4$ \\
\hline & $32 \mathrm{~A}$ & $(18 a) \alpha(19 a)^{2}(20 a)^{2}(21 a)^{2}$ & -0.939 & 2.81 & 2.78 & $3.40 / 365$ & $6.0 \times 10-2$ \\
\hline & $42 \mathrm{~A}$ & $(18 a)^{2}(19 a)^{2}(20 a)^{2}(24 a)^{\alpha}$ & -0.926 & 3.87 & 3.96 & $3.94 / 314$ & $1.6 \times 10-3$ \\
\hline
\end{tabular}

Table 2b. Configurations, Coeff., Ea, Ev, and f for the HSSO. ${ }^{a}$

\begin{tabular}{ccccccc}
\hline \multirow{2}{*}{ State } & Configuration & \multicolumn{2}{c}{ Ea(eV) } & Ev(eV/nm $)$ & $\mathrm{f}$ \\
& Occupation & Coeff. & CASSCF & CASPT2 & 0 & 0 \\
\hline $12 \mathrm{~A}$ & $(19 \mathrm{a})^{2}(20 \mathrm{a})^{2}(21 \mathrm{a})^{\alpha}$ & 0.950 & 0 & 0 & 0 & $1.3 \times 10-3$ \\
$22 \mathrm{~A}$ & $(19 \mathrm{a})^{\alpha}(20 \mathrm{a})^{2}(21 \mathrm{a})^{2}$ & 0.945 & 1.89 & 1.89 & $2.40 / 516$ & $3.8 \times 10-4$ \\
$32 \mathrm{~A}$ & $(19 \mathrm{a})^{2}(20 \mathrm{a})^{\alpha}(21 \mathrm{a})^{2}$ & -0.878 & 5.05 & 5.06 & $3.21 / 386$ & \\
& $(19 \mathrm{a}) \alpha(20 \mathrm{a})^{\alpha}(21 \mathrm{a})^{2}(22 \mathrm{a})^{\beta}$ & 0.210 & & & & \\
$42 \mathrm{~A}$ & $(19 \mathrm{a})^{2}(20 \mathrm{a})^{2}(22 \mathrm{a})^{\alpha}$ & 0.919 & 5.45 & 5.49 & $3.95 / 314$ & $1.7 \times 10-3$ \\
& $(19 \mathrm{a})^{\alpha}(20 \mathrm{a})^{2}(22 \mathrm{a})^{2}$ & 0.157 & & & & \\
\hline
\end{tabular}

a " $\alpha$ " and " $\beta$ " represent a singly occupied orbital with an up or down spin respectively.

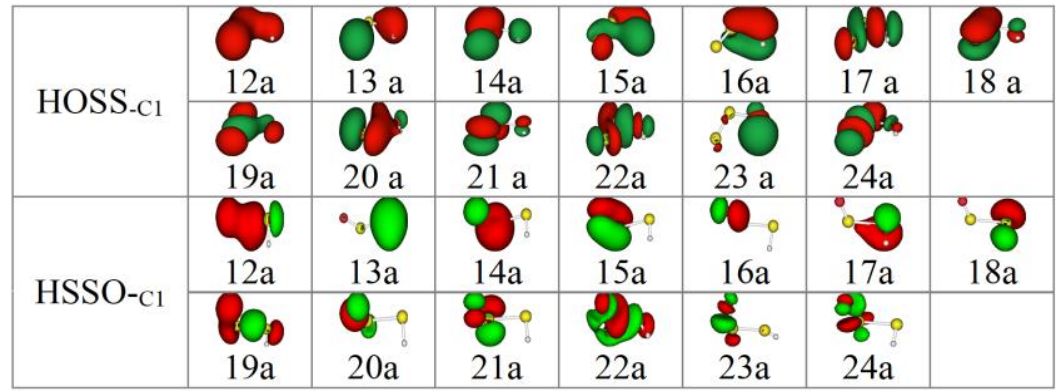

Fig.2. Plots of the occupied orbitals and vacant orbitals.

\subsection{The Harmonic Vibrational Frequencies}

Using the CASSCF/CASPT2 method, we calculated the harmonic vibrational frequencies in the gas phase for the ground and excited states of HOSS and HSSO radicals in Table $3 \mathrm{a}$ and $3 \mathrm{~b}$. And the frequencies of $\mathrm{D}_{0}$ state of TS are listed in Table $3 \mathrm{c}$.

\subsection{Reaction pathways from HOSS to HSSO on the $D_{0}$ potential energy surface}

In Figure 3, the schematic potential energy diagram of reaction pathways from HOSS to HSSO on the $\mathrm{D}_{0}$ potential energy surface is represented. According to our calculations, a transition state, with $\mathrm{C} 1$ symmetry, is included in the reaction pathway from HOSS to HSSO. The vertical ordinates are the relative values to the ground state energy. And all of the energy values of the HOSS, HSSO and TS are calculated at the CASPT2 level, using the aug-cc-pV(T+d)Z basis set. This transition state produces relatively high-energy barrier of 30.5 $\mathrm{Kcal} / \mathrm{mol}$ (and therefore big imaginary frequency) corresponding to the gradual changes about the S1-H-O angle. For the $30.5 \mathrm{kcal} / \mathrm{mol}$ energy barrier, this needs to be about $937 \mathrm{~nm}$ light to stimulate.

\section{Conclusion}

In the aug-cc-pV(n+d)Z basis sets, we gave out a combined CASSCF and CASPT2 study of the low-lying electronic states of the HOSS and HSSO radicals in C1 symmetry for the first time. The obtained geometries parameters of HOSS radical in the CASPT2/aug-cc$\mathrm{pV}(\mathrm{T}+\mathrm{d}) \mathrm{Z}$ level are closer to the values of other theoretical calculation methods for HSSO radical, the obtained bond distances and the bond angles have the same character with the ones of HOSS radical. Otherwise, the harmonic vibrational frequencies $f$, the adiabatic excitation energies Ea and the vertical excitation energies Ev were also calculated in our work firstly. Finally, we 
have given the reaction pathways in the CASPT2/aug-cc$\mathrm{pV}(\mathrm{T}+\mathrm{d}) \mathrm{Z}$ level from HOSS to HSSO on the $\mathrm{D}_{0}$ potential energy surface. In our calculations, a transition state is given during the reaction pathway from HOSS to HSSO with a relatively high-energy barrier of $30.5 \mathrm{Kcal} / \mathrm{mol}$.

Table 3a. Harmonic vibrational frequencies (in $\mathrm{cm}-1$ ) for the HOSS.

\begin{tabular}{llccccc|c}
\hline \multicolumn{1}{c}{ State } & $v_{1}\left(\mathrm{a}^{\prime}\right)$ & $v_{2}\left(\mathrm{a}^{\prime}\right)$ & $v_{3}\left(\mathrm{a}^{\prime}\right)$ & $v_{4}\left(\mathrm{a}^{\prime}\right)$ & $v_{5}\left(\mathrm{a}^{\prime}\right)$ & $v_{6}\left(\mathrm{a}^{\prime}\right)$ \\
\hline $\mathrm{X}^{2} \mathrm{~A}$ & CASPT2/aug-cc-pV(T+d)Z & 3711 & 743 & 665 & 1121 & 289 & 460 \\
& CASSCF/aug-cc-pV(T+d)Z & 3693 & 740 & 616 & 1190 & 299 & -277 \\
& {$[3540$} & 730 & 653 & 1090 & --- & --- \\
& ${ }^{[3]}$ exp. & 3512 & 737 & 652 & 1125 & --- & --- \\
$2^{2} \mathrm{~A}$ & CASp. & 3711 & 749 & 516 & 1208 & 201 & 472 \\
& CASSCF/aug-cc-pV(T+d)Z & 3743 & 780 & 534 & 1252 & 238 & 534 \\
$3^{2} \mathrm{~A}$ & CASPT2/aug-cc-pV(T+d)Z & 3517 & 750 & 423 & 1160 & 247 & 353 \\
& CASSCF/aug-cc-pV(T+d)Z & 3543 & 786 & 356 & 1225 & 231 & 262 \\
\hline
\end{tabular}

Table 3b. Harmonic vibrational frequencies (in cm-1) for the HSSO.

\begin{tabular}{llcccccc}
\hline & \multicolumn{1}{c}{ State } & $v_{1}\left(\mathrm{a}^{\prime}\right)$ & $v_{2}\left(\mathrm{a}^{\prime}\right)$ & $v_{3}\left(\mathrm{a}^{\prime}\right)$ & $v_{4}\left(\mathrm{a}^{\prime}\right)$ & $v_{5}\left(\mathrm{a}^{\prime}\right)$ & $v_{6}\left(\mathrm{a}^{\prime}\right)$ \\
\hline $\mathrm{X}^{2} \mathrm{~A}$ & CASPT2/aug-cc-pV(T+d)Z & 2758 & 1117 & 487 & 745 & 354 & 251 \\
& CASSCF/aug-cc-pV(T+d)Z & 2839 & 1134 & 530 & 878 & 330 & 168 \\
& {$[3]$ exp. } & 2560 & 1091 & 458 & --- & --- & --- \\
& ${ }^{2}$ exp. & 2560 & 1091 & 461 & --- & -- & -- \\
$2^{2} \mathrm{~A}$ & CASPT2/aug-cc-pV(T+d)Z & 2716 & 729 & 538 & 866 & 188 & 428 \\
& CASSCF/aug-cc-pV(T+d)Z & 2701 & 708 & 343 & 787 & 99 & 251 \\
$3^{2} \mathrm{~A}$ & CASPT2/aug-cc-pV(T+d)Z & 2848 & 1078 & 72 & 218 & 70 & 51 \\
& CASSCF/aug-cc-pV(T+d)Z & 2844 & 1058 & 35 & 125 & 30 & 20 \\
\hline
\end{tabular}

Table 3c. Harmonic vibrational frequencies $\left(\right.$ in $\mathrm{cm}^{-1}$ ) for the $\mathrm{D}_{0}$ state of TS.

\begin{tabular}{ccccccc}
\hline Basis set & $v_{1}\left(\mathrm{a}^{\prime}\right)$ & $v_{2}\left(\mathrm{a}^{\prime}\right)$ & $v_{3}\left(\mathrm{a}^{\prime}\right)$ & $v_{4}\left(\mathrm{a}^{\prime}\right)$ & $v_{5}\left(\mathrm{a}^{\prime}\right)$ & $v_{6}\left(\mathrm{a}^{\prime}\right)$ \\
\hline aug-cc-pV(T+d)Z & 2057 & 1230 & 1202 & 1039 & 567 & -1747 \\
\hline
\end{tabular}

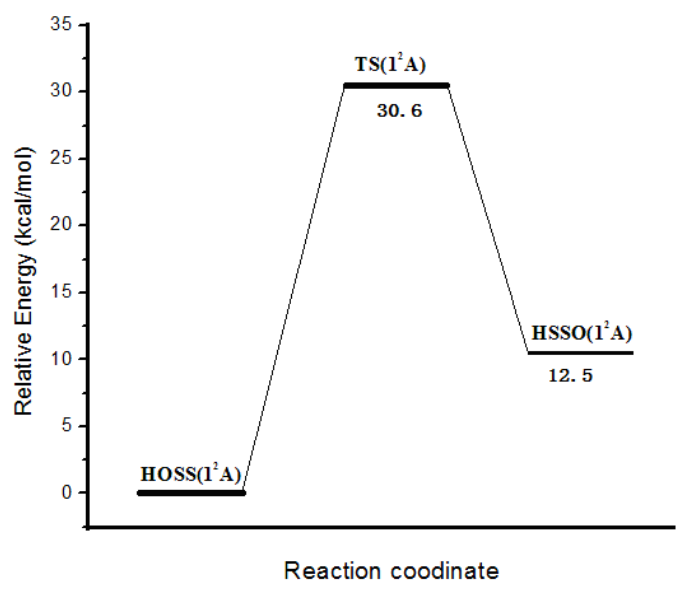

Fig. 3. $\mathrm{D}_{0}$ potential energy surface for the reaction from HOSS to HSSO at the CASPT2 level.

\section{Acknowledgment}

Authors acknowledge the funding support from national natural science foundation project of People's Republic of China(No.51141009), science and technology research project of Jiamusi university of People's Republic of China(No.Lz2013-017)and the basic scientific research operating expenses of Heilongjiang provincial universities of People's Republic of China(No. 2018KYYWF-0956) for current research.

\section{References}

1. Petris, G., Rosi, M. J Phys Chem A , 111,6526(2007).

2. Steudel, R., Steudel, Y. J Phys Chem A , 113,9920(2009).

3. Reisenauer, H. P., Mloston, G. Eur J Org Chem. 3408( 2012).

4. Malmqvist, P.-Å., Rendell, A.J. Phys. Chem., 5477(1990).

5. Andersson, K., Malmqvist, P.-Å.J. Chem. Phys. 96 , 1218(1992).

6. Malmqvist, P.-Å., Roos, B. O. Chem. Phys. Lett. $155,189(1989)$.

7. Grant, D. J., Dixon, D. A.J Phys Chem A , $113,11343(2009)$. 